\title{
Jiedu Xiaozheng Yin, a Chinese herbal formula, inhibits tumor angiogenesis via downregulation of VEGF-A and VEGFR-2 expression in vivo and in vitro
}

\author{
ZHIYUN CAO ${ }^{1}$, WEI LIN ${ }^{1}$, ZHENGRONG HUANG $^{2}$, XUZHENG CHEN $^{1}$, JINYAN ZHAO $^{1}$, \\ LIANGPU ZHENG ${ }^{1}$, HONGZHI YE ${ }^{1}$, ZHIZHEN LIU ${ }^{1}$, LIANMING LIAO ${ }^{1}$ and JIAN DU ${ }^{1}$
}

${ }^{1}$ Fujian Academy of Integrative Medicine, Fujian University of Traditional Chinese Medicine; ${ }^{2}$ Integrated Chinese and
Western Medicine Department, Fujian Provincial Cancer Hospital, Minhou Shangjie, Fuzhou, Fujian 350108, P.R. China

Received September 14, 2012; Accepted October 30, 2012

DOI: 10.3892/or.2012.2202

\begin{abstract}
Angiogenesis is crucial for cancer growth and metastasis and inhibition of angiogenesis has been recognized to be a promising strategy for the treatment of cancer. Traditional Chinese medicine (TCM) has been used for thousands of years to treat cancer. Jiedu Xiaozheng Yin (JXY), a polyherbal formula of TCM, has been used to treat various tumors in China. However, the mechanism of its anticancer activity has yet to be fully elucidated. Using human umbilical vein endothelial cells (HUVECs), chick chorioallantoic membrane (CAM) and a hepatoma mouse xenograft model, we investigated the underlying molecular mechanisms of ethanol extract of Jiedu Xiaozheng Yin (EE-JXY). EE-JXY treatment significantly inhibited tumor cell growth both in vitro and in the mouse xenograft model $(\mathrm{P}<0.05)$. Moreover, EE-JXY
\end{abstract}

Correspondence to: Dr Lianming Liao or Dr Jian Du, Fujian Academy of Integrative Medicine, Fujian University of Traditional Chinese Medicine, 1 Huatuo Road, Minhou Shangjie, Fuzhou, Fujian 350108, P.R. China

E-mail:1lm@fjtcm.edu.cn

E-mail: dujian@fjtcm.edu.cn

Abbreviations: HDW, Hedyotis Diffusa Willd; SF, Sophora flavescens; PC, Psuedobulbus Cremastrae; EE-JXY, ethanol extract of Jiedu Xiaozheng Yin; DMSO, dimethyl sulfoxide; TCM, traditional Chinese medicine; VEGF-A, vascular endothelial growth factor A; VEGFR-2, vascular endothelial growth factor receptor 2; bFGF, basic fibroblast growth factor; RPMI-1640, Roswell Park Memorial Institute medium-1640; DMEM, Dulbecco's modified Eagle's medium; FBS, fetal bovine serum; HUVECs, human umbilical vein endothelial cells; MTT, 3-(4,5-dimethylthiazol-2-yl)-2,5-diphenyltetrazolium bromide; CAM, chick chorioallantoic membrane; DAB, 3,3'-diaminobenzidine; HRP, horseradish peroxidase; RTK, receptor tyrosine kinase; TV, tumor volume; PDGFR, platelet-derived growth factor receptor; FLT-3, fms-like tyrosine kinase receptor-3

Key words: Jiedu Xiaozheng Yin, Chinese medicine, angiogenesis, vascular endothelial growth factor A, vascular endothelial growth factor receptor 2 reduced tube formation of HUVECs and angiogenesis in the CAM $(\mathrm{P}<0.01)$ and microvessel density $(\mathrm{MVD})$ of tumor in vivo $(\mathrm{P}<0.05)$. Further studies showed that EE-JXY was able to suppress the expression of vascular endothelial growth factor A (VEGF-A) and vascular endothelial growth factor receptor 2 (VEGFR-2) in both HepG2 cells and HUVECs $(\mathrm{P}<0.01)$ and in tumor $(\mathrm{P}<0.01)$. Thus, JXY suppressed tumor growth at least by inhibiting angiogenesis.

\section{Introduction}

At present, therapies for cancer mainly include surgical treatment and non-surgical therapeutic modalities such as chemotherapy and radiotherapy. Both drug resistance and toxicity limit the efficacy of non-surgical therapies. Therefore, oncologists are seeking new therapeutic approaches. Natural products, including traditional Chinese medicine (TCM), have been used for thousands of years as important remedies for a variety of diseases, including cancer. Due to their relatively fewer side-effects, there has always been an interest in the use of natural products for the treatment of cancer (1-3).

According to the theory of TCM, cancer is caused by the accumulation of foreign toxins that are harmful to the human body. For this reason, heat clearing and detoxification herbs (Qingre Jiedu herbs) such as Hedyotis Diffusa Willd (HDW), Sophora flavescens (SF), Psuedobulbus Cremastrae (PC), Prunella Bidens and Ban Zhi Lian (4-6) are commonly used to treat cancer in China. Pharmacological studies have shown that these herbs may contain ingredients that can inhibit proliferation and induce apoptosis of tumor cells (7-9).

Angiogenesis is the formation of new blood capillaries from existing vessels, supplying nutrients and oxygen to and removing waste products from cells that are distant from existing blood vessels (10). Physiological angiogenesis is involved in wound healing, reproduction and embryonic development (11). In addition to its normal physiological role, angiogenesis contributes to the pathology of a number of diseases including tumor progression and metastasis $(12,13)$. As all cells, cancer cells require a constant supply of nutrients and oxygen in order to grow and divide. Without an adequate blood supply tumors do not grow. 
The induction of angiogenesis is mediated by a variety of molecules secreted from the cells within the tumor. Vascular endothelial growth factor (VEGF) is one of the most effective biological inducers of angiogenesis. VEGF expression is upregulated by hypoxia (14) and it serves as a major angiogenic factor in vascular development in tumor (15). VEGF exhibits high affinity binding to vascular endothelial growth factor receptor (VEGFR), two distinct receptor tyrosine kinases (RTK) on endothelial cells. An essential role of VEGF in tumor angiogenesis has been demonstrated in animal models showing that neutralizing VEGF antibodies and dominant-negative VEGFR inhibit both angiogenesis and the progression of tumor (16). Angiogenesis is also related to metastasis as tumors with higher densities of blood vessels are more likely to spread. Thus, antiangiogenesis therapy is considered to be an important approach that can enhance the efficiency of chemotherapy (17). Studies have shown that some TCM herbs can inhibit angiogenesis by downregulating VEGF or VEGFR expression. For example, Li et al reported that brucine significantly reduced VEGF expression and microvessel density and inhibited the growth of breast cancer and bone metastasis in a nude mouse model (18). Shen et al reported that Pien Tze Huang suppressed the expression of VEGF-A and basic fibroblast growth factor (bFGF) at both mRNA and protein levels to inhibit tumor angiogenesis in chick chorioallantoic membrane (CAM) and human umbilical vein endothelial cells (HUVECs) (19).

Jiedu Xiaozheng Yin (JXY) is a polyherbal formula of TCM established according to the theory of Chinese medicine. It is composed of HDW (30 g), Prunella (15 g), PC (15 g) and SF (15 g). These herbs are capable of heat-clearing and detoxification. Our previous clinical studies showed that JXY can prolong the overall survival time of patients and improve their quality of life (20). We demonstrated that HDW extract may inhibit angiogenesis, induce tumor cell apoptosis by the mitochondrion-dependent pathway $(7,8)$ and inhibit proliferation of cancer cells by regulating the cell cycle (9). However, the precise mechanism of its anticancer activity remains to be fully elucidated.

In the present study, we elucidated the underlying mechanism of JXY's inhibitory effect on tumor growth. For this purpose, effects of ethanol extract of Jiedu Xiaozheng Yin (EE-JXY) on tube formation of HUVECs and angiogenesis of a CAM and hepatoma mouse xenograft model were investigated. We also explored the effects of EE-JXY on angiogenesis-related signal pathways.

\section{Materials and methods}

Reagents. Roswell Park Memorial Institute medium-1640 (RPMI-1640), Dulbecco's modified Eagle's medium (DMEM), fetal bovine serum (FBS), penicillin-streptomycin, trypsinEDTA and TRIzol reagents were purchased from Invitrogen (Carlsbad, CA, USA). The cell cycle assay kit was from BD Biosciences (San Jose, CA, USA). SuperScript II reverse transcriptase was obtained from Promega (Madison, WI, USA). The in vitro angiogenesis assay kit was purchased from Millipore (Billerica, MA, USA). Human VEGF-A and VEGFR-2 (KDR) ELISA were obtained from R\&D Systems (Minneapolis, MN, USA). All other chemicals were obtained from Sigma Chemicals (St. Louis, MO, USA).
Preparation of ethyl acetate extract from JXY. JXY is composed of HDW (30 g), Prunella (15 g), PC (15 g) and SF $(15 \mathrm{~g})$. Four herbs of JXY were purchased from Guo Yi Tang Hospital of Fujian University of Traditional Chinese Medicine (Fuzhou, China). JXY (7.5 kg) was refluxed with $75 \%$ ethanol for $2 \times 3 \mathrm{~h}$ to obtain total extract. The alcohol was removed under vacuum using a rotary evaporator. The residue was suspended in water, which was partitioned sequentially with petroleum ether, chloroform, ethanol and $\mathrm{n}-\mathrm{BuOH}$ to afford extracts. Four extracts were evaporated in vacuum and stored at $4^{\circ} \mathrm{C}$ prior to use. EE-JXY was diluted in DMSO into $200 \mathrm{mg} / \mathrm{ml}$ for in vitro experiments. For in vivo study, EE-JXY was dissolved in normal saline to a final concentration of $6 \mathrm{mg} / \mathrm{ml}$.

Cell culture. HUVECs and the human hepatoma cell line HepG2 were obtained from the American Type Culture Collection (ATCC, Manassas, VA, USA). HUVECs and HepG2 cells were grown in RPMI-1640 and DMEM, respectively. Both RPMI-1640 and DMEM were supplemented with $10 \%$ (v/v) FBS, $100 \mathrm{U} / \mathrm{ml}$ penicillin and $100 \mu \mathrm{g} / \mathrm{ml}$ streptomycin with $5 \% \mathrm{CO}_{2}$ at $37^{\circ} \mathrm{C}$ in a humidified environment.

Tumor xenograft. Sixteen male BALB/c nude mice weighing 18-22 g were injected with HepG2 cell suspension at the right flank. After 7 days, mice were randomly divided into two groups, the vehicle and the EE-JXY group. The vehicle group was given normal saline; the EE-JXY group was administered EE-JXY at a dose of $0.06 \mathrm{~g} / \mathrm{kg}$. Tumor size was measured daily and volume was calculated according to the following formula: tumor volume $\left(\mathrm{TV} ; \mathrm{mm}^{3}\right)=\mathrm{d}^{2} \mathrm{xD} / 2$, where $\mathrm{d}$ and $\mathrm{D}$ were the shortest and longest diameters, respectively. On Day 21, the tumor was excised and weighed. The animals were maintained in a pathogen-free facility $\left(23 \pm 2^{\circ} \mathrm{C}, 55 \pm 5 \%\right.$ humidity). Food and water were provided ad libitum. All procedures on treating mice were performed according to Animal Care Guidelines issued by Ministry of Science and Technology of the People's Republic of China and the Animal Care Committee of Fu Jian University of Traditional Chinese Medicine approved our protocols.

Cell viability assay. Cell viability was evaluated by the 3-(4,5-dimethylthiazol-2-yl)-2,5-diphenyltetrazolium bromide (MTT) colorimetric assay. HUVECs $\left(1 \times 10^{4}\right.$ cells/well $)$ were seeded into 96-well plates. The cells were treated with different concentrations of EE-JXY for different times. At the end of the treatment, $20 \mu \mathrm{l}$ MTT $(5 \mathrm{mg} / \mathrm{ml})$ was added to each well. After $4 \mathrm{~h}$, MTT was discarded and $100 \mu \mathrm{l}$ dimethyl sulfoxide (DMSO) was added to each well. The absorbance was measured at $490 \mathrm{~nm}$ with a microplate reader (Biotek, USA). Cell viability was calculated according to the following formula: cell viability $(\%)=$ average absorbance of EE-JXY group/average absorbance of control group x $100 \%$.

Capillary-like tube formation assay. Tube formation by HUVECs was evaluated using a commercially available angiogenesis assay kit (In Vitro Angiogenesis Assay kit, Millipore), according to the manufacturer's instructions. After HUVECs were treated with EE-JXY, cells were harvested and diluted $\left(1 \times 10^{4}\right.$ cells) in $50 \mu \mathrm{l}$ medium. The cells were then seeded onto a solid gel of basement proteins (ECMatrix gel) 
A

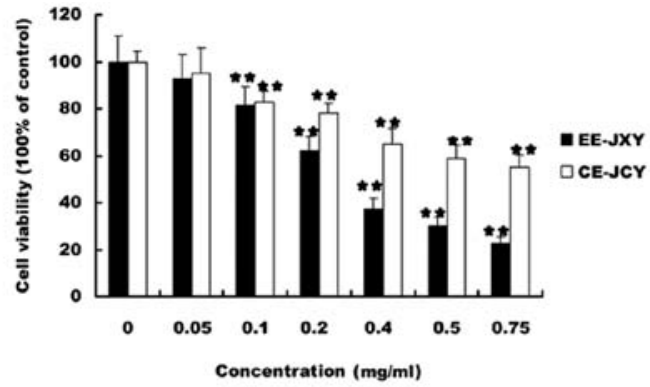

B

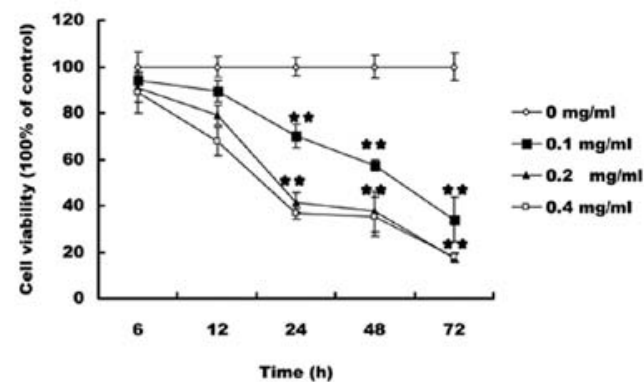

c
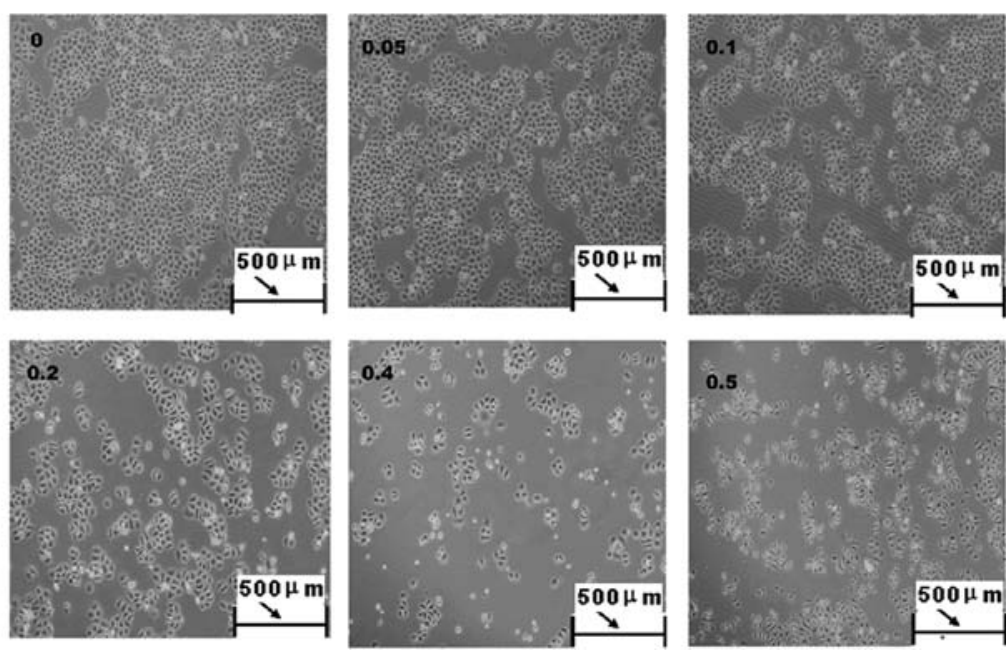

Figure 1. Effect of EE-JXY on HUVEC viability and tube formation. HUVECs were treated with the indicated concentrations of EE-JXY for the indicated times. The data were normalized to the viability of control cells (100\%, treated with $0.5 \%$ DMSO). (B) HUVECs were harvested and suspended in medium containing different concentrations of EE-JXY. The harvested cells were then seeded into basement protein-coated plates and incubated for $9 \mathrm{~h}$ at $37^{\circ} \mathrm{C}$. The development of network-like tube structures was examined by phase-contrast microscopy. The images were captured at magnification, x100. Images are representative of three independent experiments. The total length of the capillary-like tubes was measured and normalized to the untreated control. Data are the means $\pm \mathrm{SD} .{ }^{*} \mathrm{P}<0.05$ and ${ }^{* *} \mathrm{P}<0.01$, vs. control cells.

within 12-well plates and incubated for $9 \mathrm{~h}$ at $37^{\circ} \mathrm{C}$. Cellular morphology and the development of capillary tube networks were evaluated using a phase-contrast inverted microscope. Images were captured at magnification, $\mathrm{x} 100$.

VEGF-A and VEGFR-2 ELISA assay. Either HUVECs or HepG2 cells $\left(2 \times 10^{5}\right.$ cells) were seeded into 6-well plates and treated with various concentrations of EE-JXY for $24 \mathrm{~h}$. Cell supernatant was collected to measure the level of VEGF-A and cell lysates were used to determine the protein expression of VEGFR-2 in HUVECs. Measurement was performed using Quantikine ELISA kits (R\&D, USA), according to the manufacturer's instructions.

CAM assay. A CAM assay was performed to determine the in vitro anti-angiogenic activity of EE-JXY. Briefly, $10 \mu \mathrm{l}$ of EE-JXY $(10 \mathrm{mg} / \mathrm{ml})$ was loaded onto a $0.5-\mathrm{cm}$ diameter Whatman filter paper. The filter was then applied to the CAM of a 7-day embryo. Following incubation for $72 \mathrm{~h}$ at $37^{\circ} \mathrm{C}$, angiogenesis around the filter was photographed with a digital camera. The number of blood vessels in a circular perimeter surrounding the implants, at a distance of $0.25 \mathrm{~cm}$ from the edge of the filter, was counted manually.

Immunohistochemical assay. Tumor samples fixed in $10 \%$ buffered formalin for $24 \mathrm{~h}$ were processed conventionally for paraffin-embedded tumor sections. Sections were subjected to antigen retrieval and blocking of endogenous peroxidase activity. For immunostaining, sections were incubated with the primary antibodies mouse monoclonal anti-CD31 (R\&D Systems), mouse monoclonal anti-VEGFR2 (R\&D Systems) and rabbit polyclonal anti-VEGF (R\&D Systems). Sections were then incubated with biotinylated appropriate secondary antibody followed by conjugated horseradish peroxidase (HRP)streptavidin (Maixin Bio, China). Then 3,3'-diaminobenzidine (DAB; Sigma) was added, incubated at room temperature and counterstained with diluted Harris hematoxylin (Sigma). Cells were quantified by counting positive cells and total number of cells at five arbitrarily selected fields from each tumor at magnification, x100. Data are presented as percentage of positive cells.

VEGF-A and VEGFR-2 RT-PCR analysis. Total RNA was isolated with TRIzol reagent (Invitrogen) from tumor. Oligo(dT)primed RNA $(1 \mu \mathrm{g})$ was reverse-transcribed with SuperScript II reverse transcriptase (Promega), according to the manufacturer's instructions. The obtained cDNA was used to determine the amount of VEGF-A or VEGFR-2 mRNA by PCR with Taq DNA polymerase (Fermentas) using the following primers: VEGF-A forward: 5'-GCCTTGCCTTGCTGCTCTA-3', reverse: 5'-GATGTCCACCAGGGTCTCG-3'; VEGFR-2 forward: 5'-ACGCCGATTATGTGAGA-3', reverse: 5'-AGGCAGGAGT TGAGTATGT-3'; GAPDH forward: 5'-GTCATCCATGACAA CTTTGG-3', reverse: 5'-GAGCTTGACAAAGTGGTCGT-3'. 


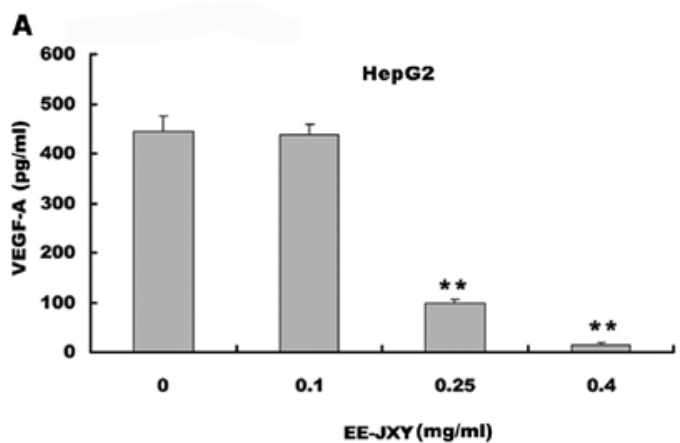

B
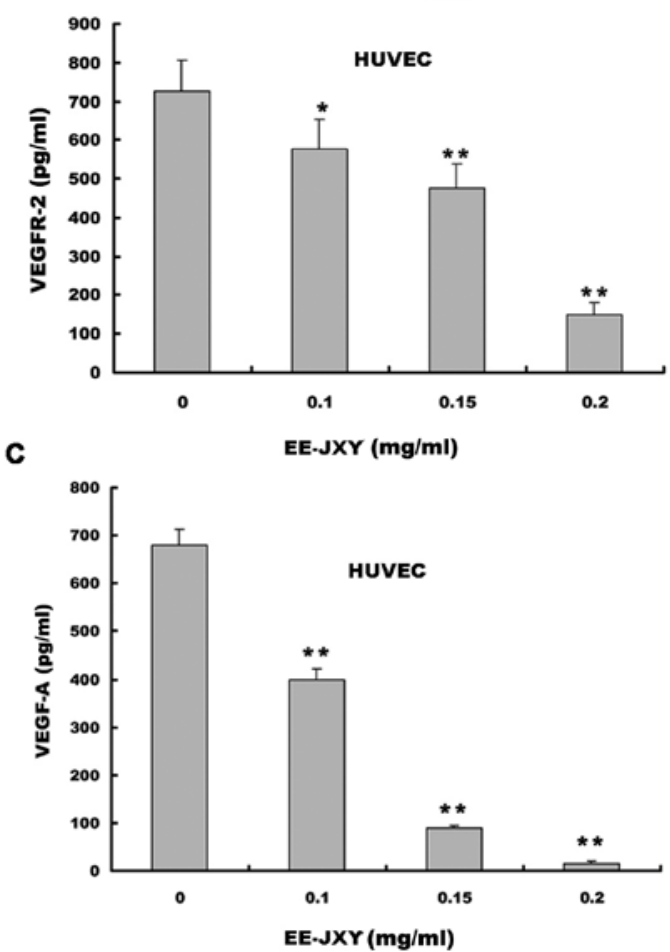

Figure 2. Effect of EE-JXY on VEGF-A and VEGFR-2 protein expression. HUVECs and HepG2 cells were treated with the indicated concentrations of EE-JXY for $24 \mathrm{~h}$. The protein levels of VEGF-A (cell culture media that is secreted) and VEGFR-2 (cell lysates) were determined by ELISA. Data are the means $\pm \mathrm{SD}$. ${ }^{*} \mathrm{P}<0.05$ and ${ }^{* *} \mathrm{P}<0.01$, vs. control cells.

Statistical analysis. All data are the means of three determinations except for the CAM assays in which 10 determinations were made for each data point. The data were analyzed using the SPSS package for Windows (Version 11.5). Statistical analysis of the data was performed with the Student's t-test and analysis of variance. Differences with $\mathrm{P}<0.05$ were considered statistically significant.

\section{Results}

EE-JXY inhibits proliferation of HUVECs. We first evaluated the effect of EE-JXY on the growth of HUVECs. HUVEC viability was determined by MTT assay following treatment with various concentrations of EE-JXY at different time-points. As shown in Fig. 1, treatment with 0.05-0.25 mg/ml of EE-JXY for 12,24 and $48 \mathrm{~h}$ reduced cell viability by $16-78 \%$, compared to untreated control cells, in a dose- and time-dependent manner ( $\mathrm{P}<0.01$; Fig. 1A).
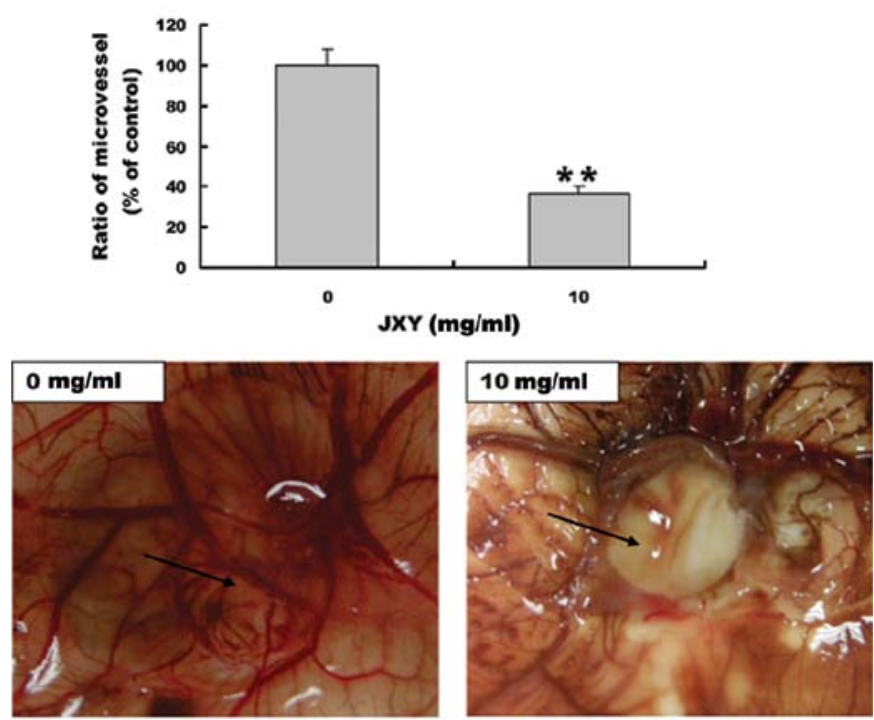

Figure 3. Effect of EE-JXY on the angiogenesis in vivo. A 0.5-cm diameter filter paper loaded with $0,0.1$ and $1 \mathrm{mg}$ of EE-JXY was applied to the chick chorioallantoic membrane and incubated at $37^{\circ} \mathrm{C}$ for $72 \mathrm{~h}$. Blood vessels surrounding the filter were photographed. Images are representative of the results obtained at each dose of EE-JXY. The number of blood vessels was quantified manually in a circular perimeter $0.25 \mathrm{~cm}$ from the edge of the filter paper inserts. Assays were performed twice with a total of 10 eggs for each tested concentration of EE-JXY. Data are the means $\pm \mathrm{SD}$. ${ }^{* *} \mathrm{P}<0.01$, vs. control.

A

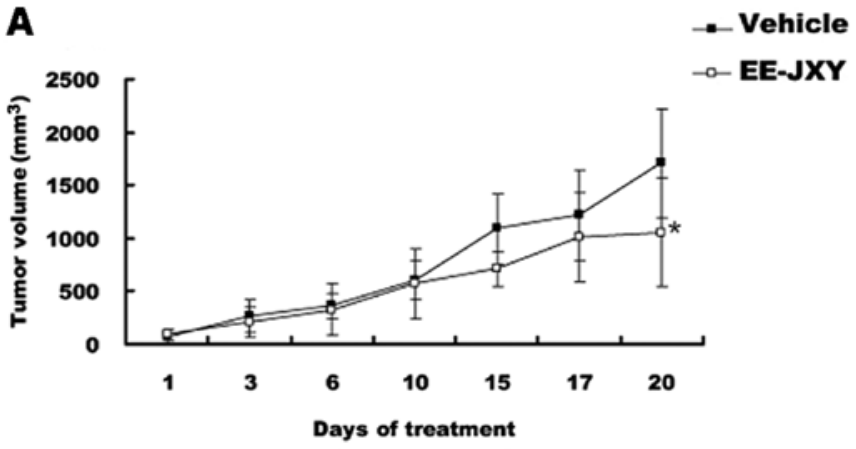

B

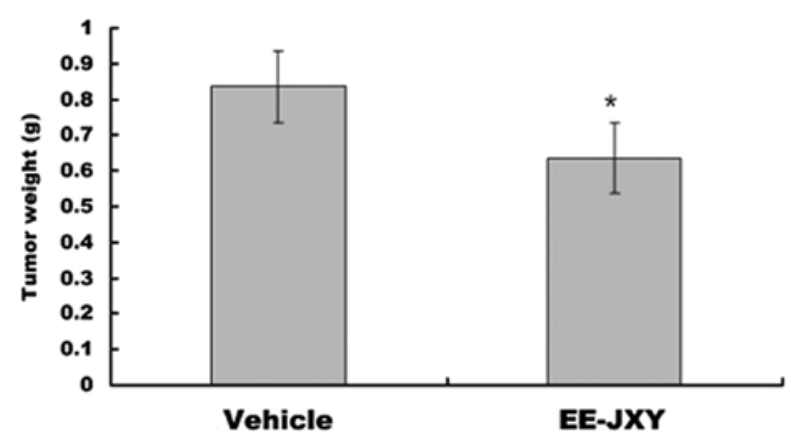

Figure 4. Effect of EE-JXY on tumor growth. Tumor bearing BALB/c nude mice were treated with either EE-JXY $(0.06 \mathrm{~g} / \mathrm{kg})$ or saline for 20 days, as described in Materials and methods. Then final tumor volume (A) and tumor weight (B) were measured. Values are the means $\pm S D, n=8$. ${ }^{*} P<0.05$, vs. vehicle group.

EE-JXY inhibits tube formation of HUVECs. To test the effect of EE-JXY on endothelial capillary tube formation, HUVECs were grown on a solid gel containing mouse basement proteins 

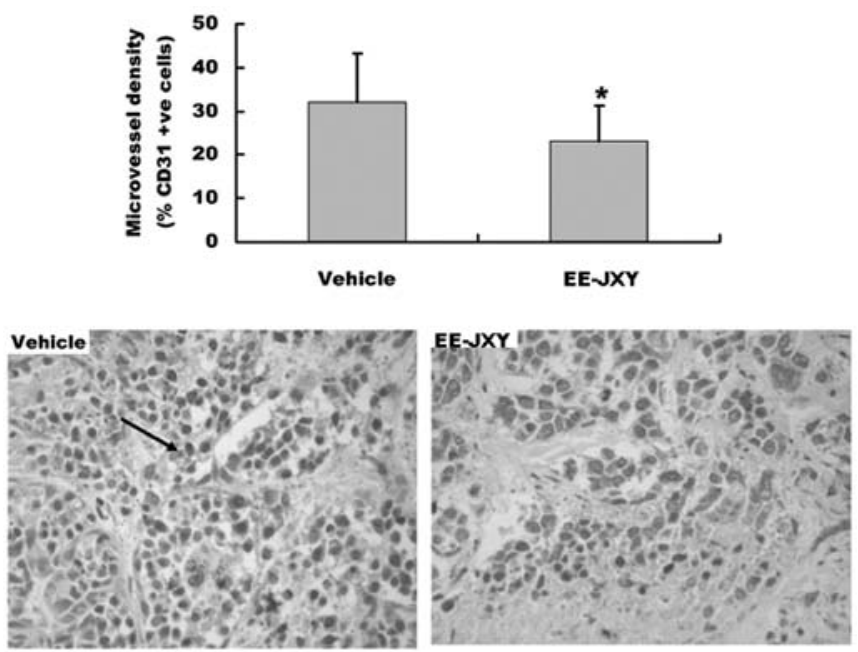

Figure 5. Effects of EE-JXY on microvessel density of the tumor. (A) Tumor bearing BALB/c nude mice were treated with either EE-JXY $(0.06 \mathrm{~g} / \mathrm{kg})$ or saline for 20 days, as described in Materials and methods. Then tumor tissue was stained for CD31. Microvessel density is shown as percentage of CD31positive cells. Values are the means $\pm \mathrm{SD}, \mathrm{n}=6$; ${ }^{*} \mathrm{P}<0.05$, vs. vehicle group. (B) Representative images of immunohistochemical staining for the control and the EE-JXY group are shown. Positive cells are indicated by arrows. Magnification, $\mathrm{x} 100$.

(ECMatrix, Millipore). This gel induces cultured endothelial cells to rapidly align and form hollow tube-like structures. As shown in Fig. 1B, untreated HUVECs formed elongated tube-like structures. By contrast, EE-JXY treatment dosedependently decreased capillary tube formation $(\mathrm{P}<0.01)$.

Effects of EE-JXY on VEGF-A and VEGFR-2 expression. We examined the effects of EE-JXY on VEGF-A secretion by HUVECs and HepG2 human hepatoma cells and on the expression of VEGFR-2 in HUVECs. The results of the ELISA assay showed that EE-JXY treatment dose-dependently reduced VEGF-A secretion by both HepG2 cells $(\mathrm{P}<0.01$; Fig. 2A) and HUVECs $(\mathrm{P}<0.01$; Fig. 2B). In addition, it suppressed VEGFR-2 expression in HUVECs ( $\mathrm{P}<0.01$; Fig. $2 \mathrm{C}$ ).

EE-JXY inhibits angiogenesis in CAM. The anti-angiogenic effect of EE-JXY was further evaluated using a classic CAM model. EE-JXY treatment significantly reduced the total number of blood vessels in the chicken embryos as compared to the untreated control $(\mathrm{P}<0.01$; Fig. 3$)$, indicating that EE-JXY is able to suppress angiogenesis in vivo.

EE-JXY inhibits tumor growth in vivo. After mice were treated with EE-JXY for 20 days, tumor volume was reduced by $39 \%$ $(\mathrm{P}<0.05)$ in the EE-JXY group compared with the vehicle group $(\mathrm{P}<0.05$; Fig. 4A). A comparison of tumor weight between the EE-JXY and the vehicle group showed a similar tendency with tumor volume ( $\mathrm{P}<0.05$; Fig. 4B).

Effect of EE-JXY on microvessel density (MVD) of tumor. We also detected tumor MVD after mice were treated with EE-JXY for 20 days, by immunohistochemical staining assay. The results showed that the MVD of the EE-JXY group was significantly lower than that of the vehicle group $(\mathrm{P}<0.05)$ (Fig. 5).
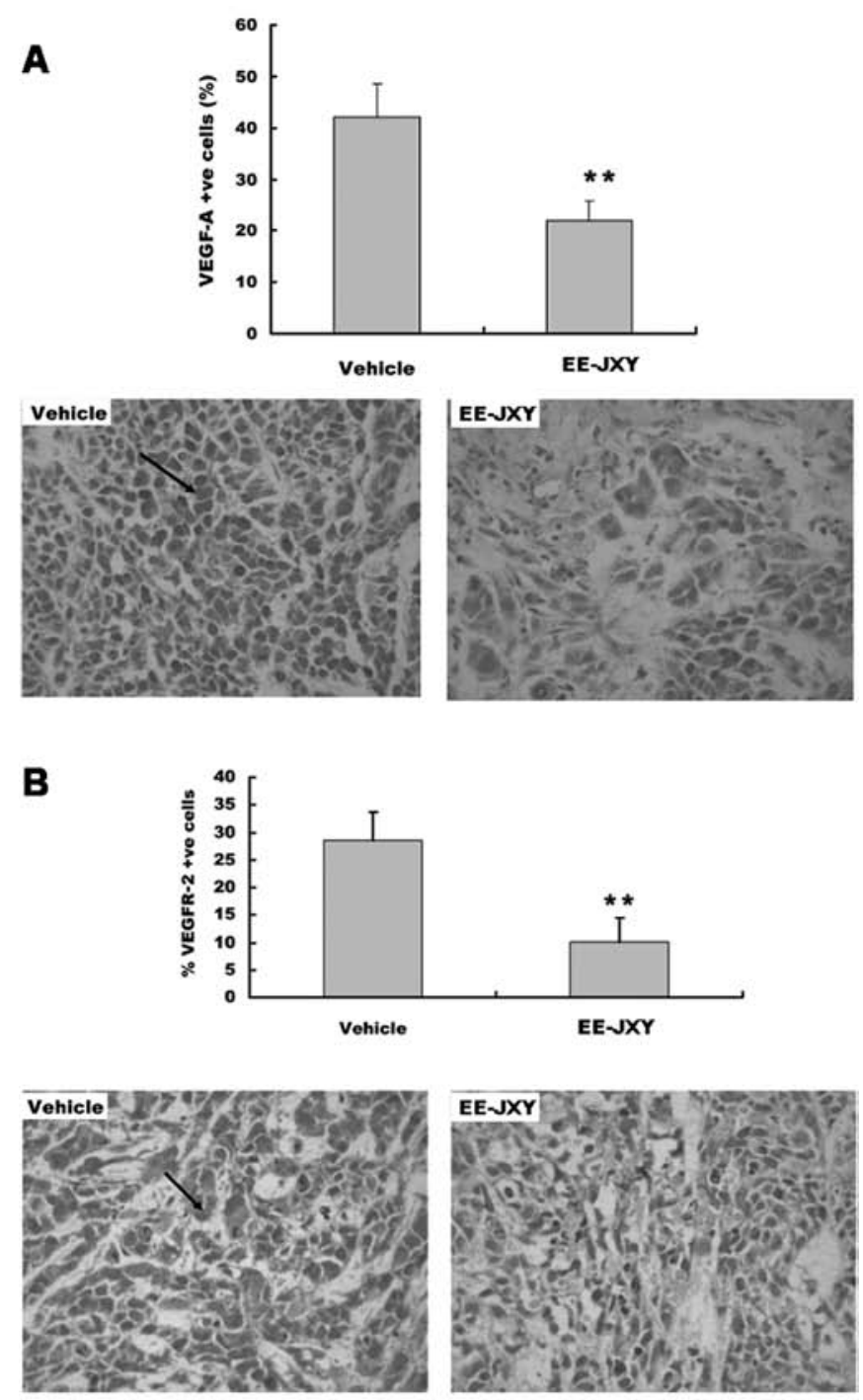

C

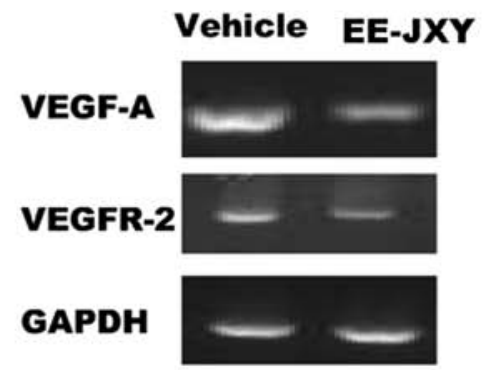

Figure 6. Effects of EE-JXY on VEGF-A and VEGFR-2 protein and gene expression. Tumor bearing BALB/c nude mice were treated with either EE-JXY $(0.06 \mathrm{~g} / \mathrm{kg})$ or saline for 20 days, as described in Materials and methods. Then, tumor tissue was stained for VEGF-A (A) and VEGFR-2 (B) and the results are shown as percentages of the positive cells. Values are the means $\pm S D, n=6 .{ }^{* *} \mathrm{P}<0.01$, vs. vehicle group. Representative images of immunohistochemical staining for VEGF-A and VEGFR-2 are shown. Positive cells are indicated by the arrow. Magnification, x100. (C) Gene expression of VEGF-A and VEGFR-2 in tumor were determined by RT-PCR. GAPDH was used as the internal control. The images are representative results of three experiments.

EE-JXY suppresses VEGF and VEGFR expression. We detected the expression of VEGF and VEGFR proteins in the tumor tissue with immunohistochemical staining. Immunohistochemistry showed there were more cells posi- 
tive for VEGF-A and VEGFR-2 in the vehicle group than in the EE-JXY group $(\mathrm{P}<0.01$; Fig. $6 \mathrm{~A}$ and $\mathrm{B})$. The results of the RT-PCR assay showed that EE-JXY treatment reduced both VEGF-A mRNA and VEGFR-2 mRNA expression in the tumor (Fig. 6C).

\section{Discussion}

Angiogenesis indicates the growth of new blood vessels from pre-existing vessels. Angiogenesis is a normal and vital process in growth and development, as well as in wound healing and in granulation tissue. However, it is also a fundamental step in the transition of tumors from a dormant to a malignant state. Tumor-associated angiogenesis allows the tumor to maintain its growth and also facilitates metastatic spreading by establishing connections to the existing vasculature (21). Therefore, targeting tumor vasculature may be as important as targeting the tumor itself. With the advent of drugs targeting the angiogenesis of cancer, patient survival has improved for several malignancies, including metastatic colorectal cancer $(22,23)$.

VEGF (including VEGF-A, VEGF-B, VEGF-C and VEGF-D) is a key regulator of physiologic angiogenesis and plays a major role in the pathobiology of cancer. VEGF-A is highly expressed in, and secreted from, various types of human cancer, and is associated with cancer progression, invasion and metastasis as well as poor patient prognosis (24). The VEGFRs are structurally related members of the RTK family that mediate critical signaling pathways in endothelial cells. Binding to VEGFR-2, VEGF starts a tyrosine kinase signaling cascade that stimulates the production of factors that variously stimulate vessel permeability (eNOS, producing NO), endothelial cell proliferation/survival (bFGF), migration (ICAMs/VCAMs/MMPs) and finally differentiation into mature blood vessels $(25,26)$.

Some TCM herbs, including Lithospermum erythrorhizon (27), Viscum album coloratum (28), Chrysobalanus icaco (29), Cassia garrettiana heartwood (30), Agaricus blazei (31), Pulsatilla koreana (32), HDW and Prunella (9) have been shown to possess anti-angiogenic activity either in vitro or in vivo. HDW and Prunella belong to heat-clearing and detoxification herbs in TCM theory. JXY is composed of four heat-clearing and detoxification herbs including HDW, Prunella, PC and SF. In the present study we found that JXY was able to inhibit tumor angiogenesis by downregulating VEGF-A and VEGFR-2 expression both in vivo and in vitro. EE-JXY decreased viability of HUVECs and their tube formation capacity. Moreover, EE-JXY inhibited angiogenesis in CAM and decreased MVD in the xenograft tumor. The above results demonstrate that JXY inhibited angiogenesis at least by downregulating VEGF-A and VEGFR-2 expression.

Targeted therapies represent a new perspective in the treatment of cancer. In contrast to conventional chemotherapy which kills both cancer cells and normal tissues, targeted drugs aim at cancer cells in a more specific manner (33). Molecules controlling cell proliferation and death, such as RTKs for growth factors, are among the best targets for this type of therapeutic approach. The era of targeted therapy began with the approval of trastuzumab, a monoclonal antibody against human epidermal growth factor receptor 2 (HER2), for the treatment of metastatic breast cancer, and imatinib, a small tyrosine kinase inhibitor targeting BCR-Abl, in chronic myeloid leukemia (34). Despite the initial enthusiasm for the efficacy of these treatments, cancer often develops resistance to trastuzumab due to the activation of alternative pathways. Similarly, in a mouse model of pancreatic islet carcinogenesis, inhibition of VEGFR2 markedly disrupted angiogenesis and initial tumor growth. However, in late-stage tumors, phenotypic resistance to VEGFR2 blockade emerged via reactivation of tumor angiogenesis, independent of VEGF, and associated with hypoxia-mediated induction of other proangiogenic factors, including members of the FGF family (35). In this view, the rationale at the basis of targeting drugs is radically shifting. There is a general agreement that molecules interfering simultaneously with multiple targets might be more effective than single target agents. Sorafenib and unitinib-targeting VEGFR, platelet-derived growth factor receptor (PDGFR), fms-like tyrosine kinase receptor-3 (FLT-3) and c-kit, are among these examples $(36,37)$.

Our previous studies demonstrated that JXY inhibits tumor growth by targeting several pathways, such as by promoting apoptosis by the mitochondrial pathway and inhibiting proliferation by the cell cycle pathway $(38,39)$. By using molecular docking simulation, Zheng et al showed that some components in HDW, Prunella and PC can combine with Bcl-xL, TNF- $\alpha$, Cdk2, IL-2 and CDK2 (40). Thus, our studies indicate that TCM formulas, composed of several herbs and therefore targeting multiple pathways (such as JXY), warrant further investigation.

\section{Acknowledgements}

This study was supported by the Chen Ke-ji Integrative Medicine Development Fund (CKJ2010020), the International Science Joint Project of the Ministry of Science and Technology of the People's Republic of China (2008DFA32200), the Key Project of Fujian Province Department of Science \& Technology (2008KJB-01) and the Fujian Province Natural Science Foundation (2012J01393). National Natural Science Foundation of China (81102582).

\section{References}

1. Zhao J, Jiang P and Zhang WD: Molecular networks for the study of TCM Pharmacology. Brief Bioinform 11: 417-430, 2010.

2. Ji HF, Li XJ and Zhang HY: Natural products and drug discovery. Can thousands of years of ancient medical knowledge lead us to new and powerful drug combinations in the fight against cancer and dementia? EMBO Rep 10: 194-200, 2009.

3. Longley DB, Allen WL and Johnston PG: Drug resistance, predictive markers and pharmacogenomics in colorectal cancer. Biochim Biophys Acta 1766: 184-196, 2006.

4. Yan Y, Cook J, McQuillan J, Zhang G, Hitzman CJ, Wang Y, Wiedmann TS and You M: Chemopreventive effect of aerosolized polyphenon $\mathrm{E}$ on lung tumorigenesis in $\mathrm{A} / \mathrm{J}$ mice. Neoplasia 9: 401-405, 2007.

5. Fang Y, Zhang Y, Chen M, Zheng $\mathrm{H}$ and Zhang K: The active component of Hedyotis diffusa Willd. Chin Trad Plant Med 26: 577-579, 2004.

6. Wu YG and Song LR: Shanghai Science and Technology Press. Zhong Hua Ben Cao 25: 530-533, 1998 (In Chinese).

7. Lin J, Wei L, Xu W, Hong Z, Liu X and Peng J: Effect of Hedyotis Diffusa Willd extract on tumor angiogenesis. Mol Med Rep 4: 1283-1288, 2011

8. Lin J, Chen Y, Wei L, Chen X, Xu W, Hong Z, Sferra TJ and Peng J: Hedyotis Diffusa Willd extract induces apoptosis via activation of the mitochondrion-dependent pathway in human colon carcinoma cells. Int J Oncol 37: 1331-1338, 2010. 
9. Chen XZ, Cao ZY, Chen TS, Zhang YQ, Liu ZZ, Su YT, Liao LM and Du J: Water extract of Hedyotis Diffusa Willd suppresses proliferation of human HepG2 cells and potentiates the anticancer efficacy of low-dose 5-fluorouracil by inhibiting the CDK2-E2F1 pathway. Oncol Rep 28: 742-748, 2012.

10. Folkman J: Tumor angiogenesis: therapeutic implications. N Engl J Med 285: 1182-1186, 1971.

11. Folkman J: Angiogenesis. Annu Rev Med 57: 1-18, 2006.

12. Fava RA, Olsen NJ, Spencer-Green G, Yeo TK, Yeo KT, Berse B, Jackman RW, Senger DR, Dvorak HF and Brown JF: Vascular permeability factor/endothelial growth factor (VPF/VEGF): accumulation and expression in human synovial fluids and rheumatoid synovial tissue. J Exp Med 180: 341-346, 1994.

13. McLaren J, Prentice A, Charmock-Jones DS, Millican SA, Muller KH, Sharkey AM and Smith SK: Vascular endothelial growth factor is produced by peritoneal fluid macrophages in endometriosis and is regulated by ovarian steroids. J Clin Invest 98: 482-489, 1996

14. Shweiki D, Itin A, Soffer D and Keshet E: Vascular endothelial growth factor induced by hypoxia may mediate hypoxia-initiated angiogenesis. Nature 359: 843-845, 1992.

15. Shalaby F, Rossant J, Yamaguchi TP, Gertsenstein M, Wu XF, Britman ML and Schuh AC: Failure of blood-island formation and vasculogenesis in Flk-1-deficient mice. Nature 376: 62-66, 1995.

16. Kim KF, Li B, Winer J, Armanin M, Gillet N, Philips HS and Ferrara N: Inhibition of vascular endothelial growth factorinduced angiogenesis suppresses tumour growth in vivo. Nature 36: 841-844, 1993.

17. Yano S, Shinohara H, Herbst RS, Kuniyasu H, Bucana CD Ellis LM, Davis DW, McConkey DJ and Fidler IJ: Expression of vascular endothelial growth factor is necessary but not sufficient for production and growth of brain metastasis. Cancer Res 60: 4959-4967, 2000.

18. Li P, Zhang M, Ma WJ, Sun X and Jin FP: Effects of brucine on vascular endothelial growth factor expression and microvessel density in a nude mouse model of bone metastasis due to breast cancer. Chin J Integr Med 18: 605-609, 2012.

19. Shen AL, Hong F, Liu LY, Lin JM, Zhuang QC, Hong ZF and Peng J: Effects of Pien Tze Huang (PTH) on angiogenesis in vivo and in vitro. Chin J Integr Med 18: 431-436, 2012.

20. Chen LW, Lin J, Chen W and Zhang WP: Effect of Chinese herbal medicine on patients with primary hepatic carcinoma in III stage during perioperational period: a report of 42 cases. Zhongguo Zhong Xi Yi Jie He Za Zhi 25: 832-834, 2005 (In Chinese).

21. Ellis LM and Hicklin DJ: VEGF-targeted therapy: mechanisms of antitumour activity. Nat Rev Cancer 8: 579-591, 2008.

22. Kerbel RS, Yu J, Tran J, Man S, Viloria-Petit A, Klement G, Coomber BL and Rak J: Possible mechanisms of acquired resistance to antiangiogenic drugs: implications for the use of combination therapy approaches. Cancer Metastasis Rev 20: 79-86, 2001.

23. Miller KD, Sweeney CJ and Sledge GW: Can tumor angiogenesis be inhibited without resistance? EXS: 95-112, 2005.

24. Carmeliet P: VEGF as a key mediator of angiogenesis in cancer. Oncology 69: 4-10, 2005.
25. Lee S, Chen TT, Barber CL, Jordan MC, Murdock J, Desai S, Ferrara N, Nagy A, Roos KP and Iruela-Arispe ML: Autocrine VEGF signaling is required for vascular homeostasis. Cell 130: 691-703, 2007.

26. Kerbel RS: Tumor angiogenesis. N Engl J Med 358: 2039-2049, 2008.

27. Hisa T, Kimura Y, Takada K, Suzuki F and Takigawa M: Shikonin: an ingredient of Lithospermum erythrorhizon, inhibits angiogenesis in vivo and in vitro. Anticancer Res 18: 783-790, 1998.

28. Yoon TJ, Yoo YC, Choi OB, Do MS, Kang TB, Lee SW, Azuma I and Kim JB: Inhibitory effect of Korean mistletoe (Viscum album coloratum) extract on tumour angiogenesis and metastasis of haematogenous and non-haematogenous tumour cells in mice. Cancer Lett 97: 83-91, 1995.

29. Alves De Paulo S, Teruszkin Balassiano I, Henriques Silva N, Oliveira Castilho R, Coelho Kaplan MA, Currie Cabral M and da Costa Carvalho MG: Chrysobalanus icaco L. extract for antiangiogenic potential observation. Int J Mol Med 5: 667-669, 2000.

30. Kim MS, Lee YM, Moon EJ, Kim SE, Lee JJ and Kim KW: Antiangiogenic activity of torilin, a sesquiterpene compound isolated from Torilis japonica. Inl J Cancer 87: 269-275, 2000.

31. Kimura Y, Baba K and Okuda H: Inhibitory effects of active substances isolated from Cassia garrettiana heartwood on tumor growth and lung metastasis in Lewis lung carcinoma-bearing mice (part 1). Anticancer Res 20: 2899-2906, 2000.

32. Takaku T, Kimura $\mathrm{Y}$ and Okuda H: Isolation of an antitumor compound from Agaricus blazei Murill and its mechanism of action. J Nutr 131: 1409-1413, 2001.

33. Casaletto JB and McClatchey AI: Spatial regulation of receptor tyrosine kinases in development and cancer. Nat Rev Cancer 12: 387-400, 2012.

34. Sastry SK and Elferink LA: Checks and balances: interplay of RTKs and PTPs in cancer progression. Biochem Pharmacol 82: 435-440, 2011.

35. Casanovas O, Hicklin DJ, Bergers G and Hanahan D: Drug resistance by evasion of antiangiogenic targeting of VEGF signaling in late-stage pancreatic islet tumors. Cancer Cell 8: 299-309, 2005

36. Faivre S, Ronot M, Dreyer C, Serrate C, Hentic O, Bouattour M, Bruno O, Couvelard A, Vilgrain V and Raymond E: Imaging response in neuroendocrine tumors treated with targeted therapies: the experience of sunitinib. Target Oncol 7: 127-133, 2012.

37. Escudier B, Szczylik C, Porta C and Gore M: Treatment selection in metastatic renal cell carcinoma: expert consensus. Nat Rev Clin Oncol 9: 327-337, 2012.

38. Zheng LP, Hu HX, Lin W, Huang YM, Zhao JY and Chen WL: The effect of serum of Jiedu Xiaozheng Yin on proliferation of gastric carcinoma cell line. Pharmacology and Clinics of Chinese Materia Medica 25: 91-93, 2009 (In Chinese)

39. Chen XZ and Du J: The effect of Jiedu Xiaozheng drink on HepG2 cells mitochondrial membrane potential. Journal of Fujian University of TCM 19: 22-23, 2009 (In Chinese).

40. Chen L, Zheng C and Du J: Study on antitumor mechanism of Qingre Xiaozheng drink by molecular docking method. Clin Pharmacol Ther 12: 324-328, 2007. 